\title{
COLEÓPTEROS EM PLANTIOS DE Eucalyptus citriodora e E. urophylla EM CUIABÁ, ESTADO DE MATO GROSSO
}

\author{
Alberto Dorval $^{1}$, Otávio Peres Filho', Eli Nunes Marques ${ }^{2}$, Rogério Goularte Moura ${ }^{3}$ \\ ${ }^{1}$ Universidade Federal de Mato Grosso, Faculdade de Engenharia Florestal, Av. Fernando Corrêa s/n, Coxipó, CEP \\ 78060-900, Cuiabá-MT, e-mail: adorval@terra.com.br.; o.peres@terra.com.br \\ 2 Pesquisador, Engenheiro Florestal, Rua Comendador Alfredo Munhoz, 345, Jardim Social, CEP 82530-110, \\ Curitiba-PR, e-mail:elinunesmarques@yahoo.com.br \\ ${ }^{3}$ Engenheiro Florestal, Doutorando em Ecologia Aplicada - ESALQ/CENA, CEP 13418-900, Piracicaba-SP, e-mail: \\ rgmoura@gmail.com
}

\section{RESUMO}

Os coleópteros foram coletados com auxílio de armadilhas de etanólicas, modelo escolitídeo/Curitiba iscadas com álcool comercial em talhões de Eucalyptus citriodora e de Eucalyptus urophylla, localizado no Município de Cuiabá, estado de Mato Grosso. Foram utilizadas seis armadilhas por talhão, distribuídas em duas linhas, com três armadilhas por linha e instaladas a 1,5 metros de altura. A distância entre as armadilhas e entre linhas foi de 30 metros. Foram coletados 4.121 e 7.290 indivíduos nos talhões de Eucalyptus citriodora e de E. urophylla, respectivamente. As famílias Scolytidade e Cerambycidae foram as mais representativas em quantidades de espécies coletadas. Cryptocarenus diadematus, C. heveae, $C$. seriatus e Hypothenemus obscurus foram as espécies mais representativas em quantidades de indivíduos coletados nos talhões das duas espécies de eucalipto. O estudo da flutuação populacional mostrou que a maioria das espécies estudadas apresentou picos populacionais nos meses do período seco na regiấo.

Palavras-chave: Coleoptera, coleobrocas, armadilha etanólica

\section{BEETLES IN PLANTATIONS OF Eucalyptus citriodora e E. urophylla IN CUIABÁ, MATO GROSSO STATE, BRAZIL}

\begin{abstract}
The beetles were collected with ethanol traps, model escolitídeo/Curitiba baited with commercial alcohol in seven years age plantations of Eucalyptus citriodora and Eucalyptus urophylla located in Cuiabá, Mato Grosso State, Brazil. Six traps were used in each plantation, distributed in two lines, with tree traps in each line and installed at $1.5 \mathrm{~m}$ of height of the soil. The distance between the traps and between the lines was 30 meters. A total of 4,121 and 7,290 individuals were collected in plantations of Eucalyptus citriodora and of E. urophylla, respectively. The families Scolytidade and Cerambycidae were the most representative in number of species collected. Cryptocarenus diadematus, C. heveae, C. seriatus and Hypothenemus obscurus were the most representative species in number of individuals collected in both Eucalyptus plantations. The data on population fluctuation indicated that most of the species studied presented population peaks during the dry season months.
\end{abstract}

Key words: Coleoptera, borer, ethanol trap 


\section{INTRODUÇÃO}

A crescente demanda de madeira tem contribuído para o aumento do número de espécies de insetos conhecidos por brocas da madeira, que causam o depreciamento da madeira pela abertura de galerias e, em algumas espécies xilomicetófagas, também pelo manchamento da madeira causado pelo fungo simbionte. Contudo, estudos científicos abordando a crescente importância destas espécies já são desenvolvidos há várias décadas. Pinheiro (1962) citou a ocorrência de nove espécies de Scolytidae, distribuídas em três gêneros, como brocas de madeira de Eucalyptus spp. Silva et al. (1968) citaram as ocorrências de Platypus dejeani em madeira de Eucalyptus tereticornis, de Platypus mutatus em madeira de Eucalyptus spp. e de Platypus rugulosus em madeira de E. citriodora.

Roberts (1969) registrou Platypus hintzi em árvores mais desenvolvidas de Eucalyptus citriodora com seis anos de idade, após um período de seca prolongada.

$\mathrm{Na}$ Tunísia de 1966 a 1972, Phoracantha semipunctata causou a morte de mais de três milhões de árvores de Eucalyptus camaldulensis, $E$. diversicolor, $E$. ovata, E. tereticornis, $E$. viminalis, $E$. maculata e $E$. gomphocephala (Chararas \& Chipoulet, 1983).

Gray (1972) citou estudos realizados em Queensland, Austrália, onde a madeira de 11 espécies de Eucalyptus apresentou alta correlação positiva entre a abundância de resina e o ataque de Lyctus brunneus (Lyctidae). Esta espécie é considerada praga de madeira de várias espécies florestais na Indonésia e na Nigéria. Minthea rugicolis (Lyctidae), espécie amplamente distribuída, é considerada praga de várias espécies de árvores nativas nas florestas tropicais da África, da Ásia e da Austrália (Gray, 1972).

- Moraes \& Berti Filho (1974) registraram a ocorrência de Eurymerus eburioides em madeira de Eucalyptus citriodora, Oxymerus nigricornis em madeira de E. tereticornis, Trachyderes thoracicus (Cerambycidae) em madeira de E. viminalis e Psiloptera doncheri (Buprestidae) em plantios de E. urophylla.

Macedo (1975) registrou a ocorrência de Xyleborus affinis e Xyleborus ferrugineus atacando árvores de E. urophylla e de $E$. saligna em várias localidades no estado de São Paulo.

Browne et al. (1976) observaram o ataque de Austroplatypus incompertus (Platypodidae) em madeira de 13 espécies de Eucalyptus spp., iniciando a abertura das galerias em árvores vivas, com troncos de casca áspera, sendo constatada uma rede ramificada de túneis sem interligações no interior da madeira.

Bigger (1982) citou a larva de Oxymagis horni (Cerambycidae) causando danos em madeira de E. deglupta nas Ilhas Solomon, sendo que os danos mais significativos ocorreram em árvores com até dois anos de idade, encontrando-se galerias com até $10 \mathrm{~cm}$ de comprimento.

A introdução de várias espécies de eucalipto em algumas regiões da Itália, para serem exploradas comercialmente e, também, para serem usadas como quebra-vento, serviu para a introdução de Phoracantha semipunctata, a mais temível praga do eucalipto (Jacoboni, 1982). As larvas de Phoracantha semipunctata penetram até o floema, alimentando-se da parte entre o floema e xilema, abrindo galerias profundas e com o secamento do floema, penetram no xilema para escapar das condições desfavoráveis, danificando-o pela abertura de galerias de até $40 \mathrm{~cm}$ de comprimento, podendo chegar a 10 $\mathrm{cm}$ de profundidade (Chararas \& Chipuilet, 1983). 
Scriven et al. (1986) afirmaram que a susceptibilidade ao ataque de Phoracantha semipunctata é variável entre as espécies de eucalipto estressadas pela seca, com os ataques maciços matando rapidamente árvores de $E$. globulus e de E. viminalis, principalmente quando suas defesas estão baixas, enquanto que, nas mesmas condições, em E. blakelyi, ocorreu alta mortalidade de larvas em galerias sob a casca, entretanto algumas larvas sobrevivem e causaram grandes lesões às árvores.

No Brasil, a introdução de Phoracantha semipunctata iniciou-se pela região Sul em 1956 (Biezanko \& Bosq, 1956), sendo detectado no estado de São Paulo em plantios de E. citriodora em 1984 (Berti Filho, 1997).

A ocorrência de Astraeus mastersi (Buprestidae) em madeira de $E$. propinqua e de $E$. maculata por Calymmaderus incisus (Anobiidae) em residências de Queensland e Brisbane (Austrália) e a conclusão de que as larvas poderiam completar seu ciclo em madeira de Pinus spp. foram observadas por Hawkeswood (1986) e Hockey (1986).

Ohmart \& Edwards (1991) afirmaram que um complexo de seis a dez espécies de Scolytidae, Platypodidae e Bostrichidae são responsáveis pela maioria dos danos ocorridos em árvores e madeira de Eucalyptus spp. no Brasil.

Os insetos broqueadores reproduzem-se mais rapidamente em povoamentos maduros, porque o vigor e a capacidade de defesa destes povoamentos diminuem com o avanço da idade (Disperati, 1995).

No Chile, o setor florestal, após a introdução acidental das espécies Hylugus ligniperda e Hylastes ater (Scolytidae), vetores do fungo Ceratocystes pilifera causador da mancha azul na madeira, aumentou a preocupação com a qualidade de produtos oriundos da madeira e destinados à exportação (Mayorga, 2000).

O objetivo deste trabalho é fornecer subsídios para o conhecimento das espécies de coleópteros que ocorrem associadas aos plantios florestais de Eucalyptus citriodora e de Eucalyptus urophylla no município de Cuiabá, estado de Mato Grosso.

\section{MATERIAL E MÉTODOS}

Os levantamentos foram realizados na Fazenda Mutuca, localizada no município de Cuiabá, estado de Mato Grosso entre as latitudes $15^{\circ} 10^{\prime}$ Sul e longitude $56^{\circ} 40^{\prime}$ Oeste. O clima é do tipo AW (Köppen), abrangendo toda a depressão cuiabana, sendo caracterizado como tropical de altitude de planalto, com chuvas de verão. A temperatura média mensal da região é de $25,7^{\circ} \mathrm{C}$ e a precipitação pluviométrica é de $1.400 \mathrm{~mm}$ anual, concentrando-se no período compreendido entre os meses de novembro a abril.

O levantamento populacional foi realizado em talhões de Eucalyptus citriodora e de Eucalyptus urophylla, implantados em 1991. Foi amostrado um talhão de cada espécie de eucalipto. Os insetos foram coletados com auxílio de armadilha etanólica, modelo escolitídeo/Curitiba, iscadas com álcool comercial, sendo utilizadas seis armadilhas por talhão, distribuídas em duas linhas, com três armadilhas por linha e instaladas a 1,5 metros de altura. À distância entre as armadilhas e entre as linhas foi de 30 metros.

A análise quantitativa foi feita mediante a contagem direta dos exemplares. Foram calculados os índices faunísticos de constância (Dajóz,1974), dominância (Sakagami \& Matsumura, 1967), abundância (Silveira Neto et al., 1976) e de diversidade (Ludwig \& Reynolds, 1988). Para as espécies que apresentaram freqüencias de capturas superiores a $5 \%$ e que ocorreram na análise faunística como constantes, muito abundantes e dominantes foram realizados estudos de flutuação populacional e de correlação com as 
temperaturas mínima, média, máxima, umidade relativa e precipitação pluvial.

\section{RESULTADOS E DISCUSSÃO}

No talhão de E. citriodora foram capturados 4.121 indivíduos, distribuídos em 28 gêneros e 43 espécies. As famílias Scolytidae com 11 gêneros $(30,21 \%)$ e 26 espécies $(60,47 \%)$ e Cerambycidae com sete gêneros $(25 \%)$ e sete espécies $(16,28 \%)$ foram as mais representativas. Scolytidae com 3.869 indivíduos coletados $(93,69 \%)$ foi a mais importante (Tabela 1).

TABELA 1. Quantidades (QT) e percentagens (\%) de gêneros, espécies e de espécimens coletados com armadilhas etanólicas em talhões de Eucalyptus citriodora. Cuiabá, MT, março/1998 a fevereiro/1999.

\begin{tabular}{lcccccc}
\hline \multirow{2}{*}{ Família } & \multicolumn{2}{c}{ Gêneros } & \multicolumn{2}{c}{ Espécies } & \multicolumn{2}{c}{ Espécimens } \\
\cline { 2 - 7 } & QT & $\%$ & QT & $\%$ & QT & 0,34 \\
Anthribidae & 01 & 3,57 & 01 & 2,33 & 14 & 3,57 \\
Bostrychidae & 03 & 10,71 & 03 & 6,98 & 147 & 0,02 \\
Bruchidae & 01 & 3,57 & 01 & 2,33 & 01 & 0,70 \\
Cerambycidae & 07 & 25,00 & 07 & 16,28 & 29 & 0,12 \\
Curculionidae & 03 & 10,71 & 03 & 6,98 & 05 & 0,05 \\
Elateridae & 01 & 3,57 & 01 & 2,33 & 02 & 1,31 \\
Platypodidae & 01 & 3,57 & 01 & 2,33 & 54 & 93,69 \\
Scolytidae & 11 & 30,29 & 26 & 60,47 & 3.869 & 100 \\
\hline Total & 28 & 100 & 43 & 100 & 4.121 & \\
\hline
\end{tabular}

No talhão de E. urophylla foram capturados 7.290 indivíduos distribuídos em 35 gêneros e 59 espécies (Tabela 2). As famílias Scolytidae com 12 gêneros $(34,29 \%)$ e 33 espécies $(55,93 \%)$, Cerambycidae com dez gêneros $(28,57 \%)$ e 12 espécies $(20,34 \%)$ e Bostrichidae com cinco gêneros $(14,29 \%)$ e cinco espécies $(8,47 \%)$ foram as mais importantes. Scolytidae com 6.537 indivíduos $(89,67 \%)$ coletados foi a mais representativa (Tabela 2 ).

TABELA 2. Quantidades (QT) e percentagens (\%) de gêneros, espécies e de espécimens coletados com armadilhas etanólicas em talhões de Eucalytptus urophylla. Cuiabá, MT, março/1998 a fevereiro/1999.

\begin{tabular}{|c|c|c|c|c|c|c|}
\hline \multirow[b]{2}{*}{ Família } & \multicolumn{2}{|c|}{ Gêneros } & \multicolumn{2}{|c|}{ Espécies } & \multicolumn{2}{|c|}{ Espécimens } \\
\hline & QT & $\%$ & QT & $\%$ & QT & $\%$ \\
\hline Anthribidae & 01 & 2,86 & 01 & 1,69 & 14 & 0,19 \\
\hline Bostrychidae & 05 & 14,29 & 05 & 8,47 & 277 & 3,80 \\
\hline Bruchidae & 01 & 2,86 & 01 & 1,69 & 03 & 0,04 \\
\hline Cerambycidae & 10 & 28,57 & 12 & 20,34 & 53 & 0,73 \\
\hline Curculionidae & 03 & 8,57 & 03 & 5,08 & 06 & 0,08 \\
\hline Elateridae & 02 & 5,71 & 02 & 3,39 & 19 & 0,26 \\
\hline Platypodidae & 01 & 2,86 & 02 & 3,39 & 381 & 5,23 \\
\hline Scolytidae & 12 & 34,29 & 33 & 55,93 & 6.537 & 89,67 \\
\hline Total & 35 & 100 & 59 & 100 & 7.290 & 100 \\
\hline
\end{tabular}

Ocorreram 31 espécies de coleópteros comuns aos talhões das duas espécies de eucaliptos, 13 espécies restritas a $E$. citriodora e 27 que ocorreram somente no talhão de E. urophylla. Cryptocarenus diadematus, $C$. heveae, $C$. seriatus, Hypothenemus eruditus, H. obscurus e Xyleborus retusus (Scolytidae) apresentaram as 
maiores quantidades de indivíduos coletados no talhão de E. citriodora, enquanto que em $E$. urophylla, $C$. diadematus, $C$. heveae, $C$. seriatus, $H$. obscurus, Xyleborus spinosulus (Scolytidae) e Platypus linearis (Platypodidae) foram as espécies mais importantes (Tabela 3). Dorval \& Peres Filho (2001) registraram, em vegetação de cerrado, as ocorrências de H. obscurus, $C$. heveae, $C$. diadematus, $C$. seriatus e
$H$. eruditus como as mais representativas em números de indivíduos coletados. Apesar de terem sido coletadas várias espécies de Cerambycidae com potencial para danos em várias espécies florestais, estas espécies ocorreram com poucos indivíduos. Dentre os cerambicídeos coletados, $A$. surinamum e $N$. pusillus já foram citados como brocas de madeira de Eucalyptus viminalis, E. citriodora (Moraes \& Berti Filho, 1974) e E. pellita (Zanuncio et al., 1993).

TABELA 3. Famílias, Espécies e quantidades de indivíduos coletados com armadilhas etanólicas em talhões de Eucalyptus citriodora e de Eucalyptus urophylla. Cuiabá, MT, março/1998 a fevereiro/1999.

\begin{tabular}{|c|c|c|}
\hline Família/Espécie & $\begin{array}{l}\text { Eucalyptus } \\
\text { citriodora }\end{array}$ & $\begin{array}{l}\text { Eucalyptus } \\
\text { urophylla }\end{array}$ \\
\hline \multicolumn{3}{|l|}{ Anthribidae } \\
\hline Phaenithon curvipes (Germar, 1824) & 14 & 14 \\
\hline \multicolumn{3}{|l|}{ Bostrychidae } \\
\hline Bostrychopsis uncinata (Germar, 1824) & 36 & 95 \\
\hline Micrapate brasiliensis Lesne, 1898 & 80 & 117 \\
\hline Rizopertha dominica (Fabricius, 1792) & & 5 \\
\hline Xyloprista exacantha (Fairmaire, 1892) & & 3 \\
\hline Xyloperthella picea (Olivier, 1790) & 31 & 57 \\
\hline \multicolumn{3}{|l|}{ Bruchidae } \\
\hline Pachymerus nucleorum (Fabricius, 179) & 1 & 3 \\
\hline \multicolumn{3}{|l|}{ Cerambycidae } \\
\hline Acanthoderes nigricans Leméere, 1885 & & 1 \\
\hline Acanthoderes sp.1 & & 1 \\
\hline Acryson surinamum (Linné, 1767) & 1 & 11 \\
\hline Aegoschema monilefer & & 1 \\
\hline Aerenea brunea Thomson, 1868 & & 1 \\
\hline Anelaphus sp. & & 1 \\
\hline Ataxia operana & 1 & \\
\hline Brasilianus sp. & 1 & \\
\hline Chlorida festiva (Linné, 1758) & 4 & 6 \\
\hline Dorcacerus barbatus (Olivier, 1790) & & 1 \\
\hline Eburodacrys sp. & 1 & \\
\hline Estola sp. & & 1 \\
\hline Lypsimena sp. & & 1 \\
\hline Neoclytus pusillus (Laport \& Gory, 1835) & 20 & 26 \\
\hline Oreodera quinquetuberculata (Drapier,1820) & 1 & \\
\hline Trachyderes succintus (Linné,1758) & & 1 \\
\hline \multicolumn{3}{|l|}{ Curculionidae } \\
\hline Heilipodus naevulus Mannerheim, 1836 & 3 & 3 \\
\hline Naupactus sp. & 1 & \\
\hline Rhinostomus barbirostris (Fabricius, 1775) & & 1 \\
\hline Zygops sp. & 1 & 2 \\
\hline
\end{tabular}




\begin{tabular}{lcc}
\hline Família/Espécie & $\begin{array}{c}\text { Eucalyptus } \\
\text { citriodora }\end{array}$ & $\begin{array}{c}\text { Eucalyptus } \\
\text { urophylla }\end{array}$ \\
\hline Elateridae & & 3 \\
Pherhimius fascicularis (Fabricius, 1787) & 2 & 16 \\
Phyrophorus sp. & & 378 \\
Platypodidae & 54 & 3 \\
Platypus linearis Chapuis, 1865 & & 3 \\
Platypus sp. & 1 & 3 \\
Scolytidae & & 3 \\
Amphicranus sp. & & \\
Corthylus nudipennis Schedl, 1950 & & \\
Corthylocurus sp. & & \\
\hline
\end{tabular}

\begin{tabular}{|c|c|c|}
\hline \multicolumn{3}{|l|}{ Scolytidae } \\
\hline Cnesinus dryographus Schedl, 1951 & & 5 \\
\hline Cnesinus sp. & 1 & \\
\hline Cryptocaremus diadematus Eggers, 1937 & 630 & 1.198 \\
\hline Cryptocaremus heveae (Hagedorni, 1912) & 868 & 980 \\
\hline Cryptocaremus seriatus Eggers, 1933 & 768 & 1.441 \\
\hline Cryptocaremus sp. & 3 & 133 \\
\hline Hypothenemus bolivianus Eggers, 1931 & 49 & 17 \\
\hline Hypothenemus elephas Eichhoff, 1868 & 9 & 21 \\
\hline Hypothenemus eruditus Westwood, 1836 & 221 & 240 \\
\hline Hypothenemus obscurus (Fabricius, 1801) & 570 & 774 \\
\hline Hypothenemus sp.1 & 53 & 57 \\
\hline Hypothenemus sp.2 & 145 & 113 \\
\hline Hypothenemus sp.3 & 5 & 1 \\
\hline Microcorthylus minimus Sched1, 1850 & & 30 \\
\hline Microcorthylus sp. & 1 & \\
\hline Monarthum glabriculum & & 1 \\
\hline Monarthum sp. & 1 & \\
\hline Premnobius cavipennis Schedl, 1878 & 17 & 71 \\
\hline Sampsonius dampfi Schedl, 1940 & 2 & 17 \\
\hline Tricolus pernanulus Schedl, 1939 & & 1 \\
\hline Tricolus sp. & 1 & \\
\hline Xyleborus affinis Eichhoff, 1867 & 32 & 122 \\
\hline Xyleborus brasiliensis Eggers, 1928 & 2 & 3 \\
\hline Xyleborus bisseriatus Schedl, 1963 & & 1 \\
\hline Xyleborus ferruguneus (Fabricius, 1801) & 33 & 193 \\
\hline Xyleborus gracillis (Eichhoff, 1868) & 1 & \\
\hline Xyleborus hagedorni Iglesias, 1914 & 1 & \\
\hline Xyleborus obtruncatus Schedl, 1949 & & 1 \\
\hline Xyleborus paraguayensis Schedl, 1949 & & 1 \\
\hline Xyleborus retusus Eichhoff, 1868 & 224 & 154 \\
\hline Xyleborus sentosus Eichhoff, 1868 & 1 & \\
\hline Xyleborus spinosulus Schedl, 1934 & 226 & 946 \\
\hline Xyleborus truncatelus Schedl, 1949 & & 1 \\
\hline Xyleborus sp. 1 & & 2 \\
\hline Xyleborus sp.2 & & 1 \\
\hline Xyleborus sp.3 & & 1 \\
\hline Xylosandrus compactus (Eichhoff, 1875) & 4 & 4 \\
\hline Xyleborinus reconditus (Schedl, 1963) & & 1 \\
\hline Total & 4.121 & 7.289 \\
\hline
\end{tabular}


No talhão de E. citriodora, $C$. heveae, $C$. seriatus, $C$. diadematus, $H$. obscurus, H. eruditus (Scolytidae) foram as espécies com as maiores freqüências de coletas. No período ocorreram sete espécies muito abundantes, oito comuns, quatro dispersås e 24 raras. Quanto à constância, oito espécies foram constantes, três acessórias e 32 acidentais. No total, 21 espécies foram dominantes e 22 não dominantes. Apenas, $C$. diadematus, $C$. heveae, $C$. seriatus e $H$. obscurus (Scolytidae) ocorreram como muito abundantes, constantes e dominantes (Tabela 4). Dorval (2002) registrou em talhão de Eucalyptus camaldulensis ocorrências de $C$. diadematus, $C$. heveae, $C$. seriatus, $H$. obscurus e $C$. eruditus como muito abundantes, constantes e dominantes.
No talhão de E. urophylla, C. heveae, $C$. seriatus, $C$. diadematus, $X$. spinosulus, $H$. obscurus (Scolytidae) e $P$. linearis (Platypodidae) foram as espécies com as maiores freqüências de coletas. No período ocorreram sete espécies muito abundantes, duas abundantes, cinco comuns, 11 dispersas e 34 raras. Quanto a constância, oito espécies foram constantes, oito acessórias e 43 acidentais. Do total 26 espécies foram dominantes e 33 não dominantes. Somente as espécies $C$. diadematus, $C$. heveae, $C$. seriatus, $H$. obscurus, $X$. spinosulus (Scolytidae) e $P$. linearis (Platypodidae) ocorreram neste talhão como muito abundantes, constantes e dominantes (Tabela 4). Dorval (2002) observou em talhão de $E$. pellita a ocorrência das espécies $C$. diadematus, $C$. heveae, $C$. seriatus, $C$. eruditus, $H$. obscurus e $X$. spinosulus

TABELA 4. Relação das freqüências $(\mathrm{F} \%)$ e dos índices de abundância $(\mathrm{A})$, constância (C), e dominância (D) das espécies coletadas com armadilhas etanólicas em plantios de Eucalyptus citriodora e de Eucalyptus urophylla. Cuiabá, MT, março/1998 a fevereiro/1999.

\begin{tabular}{|c|c|c|c|c|c|c|c|c|}
\hline \multirow[b]{2}{*}{ Família/Espécie } & \multicolumn{4}{|c|}{ Eucalyptus citriodora } & \multicolumn{4}{|c|}{ Eucalyptus urophylla } \\
\hline & $F(\%)$ & A & $\mathrm{C}$ & $\mathrm{D}$ & $\mathrm{F}(\%)$ & $\mathrm{A}$ & $\mathrm{C}$ & $\bar{D}$ \\
\hline Anthribidae & & & & & & & & \\
\hline Phaenithon curvipes & 0,34 & d & $\mathrm{z}$ & $\mathrm{d}$ & 0,19 & d & $\mathrm{z}$ & d \\
\hline Bostrychidae & & & & & & & & \\
\hline Bostrychopsis uncinata & 0,87 & $\mathrm{c}$ & $\mathrm{w}$ & d & 1,30 & $\mathrm{c}$ & $\mathrm{y}$ & $d$ \\
\hline Micrapate brasiliensis & 1,94 & $d$ & $\mathrm{y}$ & $d$ & 1,60 & $\mathrm{c}$ & y & $\mathrm{d}$ \\
\hline Rizopertha dominica & & & & & 0,07 & $\mathrm{r}$ & $\mathrm{z}$ & nd \\
\hline Xyloprista exacantha & & & & & 0,04 & $\mathrm{r}$ & $\mathrm{z}$ & nd \\
\hline Xyloperthella picea & 0,75 & $\mathrm{c}$ & $\mathrm{z}$ & $\mathrm{d}$ & 0,78 & $\mathrm{~d}$ & $\mathrm{y}$ & $\mathrm{d}$ \\
\hline Bruchidae & & & & & & & & \\
\hline Pachymerus nucleorum & 0,02 & $\mathrm{r}$ & $\mathrm{z}$ & nd & & & & \\
\hline Cerambycidae & & & & & & & & \\
\hline Acanthoderes nigricans & & & & & 0,01 & $\mathrm{r}$ & $\mathrm{z}$ & nd \\
\hline Acanthoderes sp.1 & & & & & 0,01 & $\mathrm{r}$ & $\mathrm{z}$ & nd \\
\hline Achryson surinamum & 0,02 & $\mathrm{r}$ & $\mathrm{z}$ & nd & 0,01 & $\mathrm{~d}$ & $\mathrm{z}$ & d \\
\hline Aegoschema monilefer & & & & & 0,01 & $r$ & $\mathrm{z}$ & nd \\
\hline Aerenea brunea & & & & & 0,01 & $\mathrm{r}$ & $\mathrm{z}$ & nd \\
\hline Anelaphus sp. & & & & & 0,01 & $\mathrm{r}$ & $\mathrm{z}$ & nd \\
\hline Ataxia operana & 0,02 & $\mathrm{r}$ & $\mathrm{z}$ & nd & & & & \\
\hline Brasilianus sp. & 0,02 & $r$ & $\mathrm{z}$ & nd & & & & \\
\hline Chlorida festiva & 0,10 & $\mathrm{r}$ & $\mathrm{z}$ & nd & 0,08 & $\mathrm{r}$ & $\mathrm{z}$ & $d$ \\
\hline Dorcacerus barbatus & & & & & 0,01 & $\mathrm{r}$ & $\mathrm{z}$ & nd \\
\hline Eburodacrys sp. & 0,02 & $\mathrm{r}$ & $\mathrm{z}$ & nd & & & & \\
\hline Estola sp. & & & & & 0,01 & $\mathrm{r}$ & $\mathrm{z}$ & nd \\
\hline Lypsimena sp. & & & & & 0,01 & $\mathrm{r}$ & $\mathrm{z}$ & nd \\
\hline
\end{tabular}




\begin{tabular}{|c|c|c|c|c|c|c|c|c|c|}
\hline \multirow[b]{2}{*}{ Família/Espécie } & \multicolumn{4}{|c|}{ Eucalyptus citriodora } & & \multicolumn{4}{|c|}{ Eucalyptus urophylla } \\
\hline & $\mathrm{F}(\%)$ & $\mathrm{A}$ & $\mathrm{C}$ & $\mathrm{D}$ & & $\mathrm{F}(\%)$ & $\mathrm{A}$ & $\mathrm{C}$ & $\mathrm{D}$ \\
\hline Neoclytus pusillus & 0,4 & & & $\mathrm{z}$ & $\mathrm{d}$ & 0,36 & $\mathrm{~d}$ & $\mathrm{z}$ & $\mathrm{d}$ \\
\hline Oreodera quinquetuberculata & 0,0 & & & $\mathrm{z}$ & nd & & & & \\
\hline Trachyderes succintus & & & & & & 0,01 & $\mathrm{r}$ & $\mathrm{z}$ & nd \\
\hline Curculionidae & & & & & & & & & \\
\hline Heilipodus naevulus & 0,07 & $\mathrm{r}$ & $\mathrm{z}$ & nd & & 0,04 & $\mathbf{r}$ & $\mathrm{z}$ & nd \\
\hline Naupactus sp. & 0,02 & $\mathrm{r}$ & $\mathrm{z}$ & nd & & & & & \\
\hline Rhinostomus barbirostris & & & & & & 0,01 & $\mathrm{r}$ & $\mathrm{z}$ & nd \\
\hline $\begin{array}{l}\text { Zygops sp. } \\
\text { Elateridae }\end{array}$ & 0,02 & $\mathrm{r}$ & $\mathrm{z}$ & nd & & 0,03 & r & $\mathrm{z}$ & nd \\
\hline Pherhimius fascicularis & & & & & & 0,04 & $\mathrm{r}$ & $\mathrm{z}$ & nd \\
\hline Phyrophorus sp. & 0,28 & $\mathrm{r}$ & $\mathrm{z}$ & d & & 0,22 & $\mathrm{c}$ & $\mathrm{z}$ & $\mathrm{d}$ \\
\hline Platypodidae & & & & & & & & & \\
\hline Platypus linearis & 2,81 & $\mathrm{c}$ & $\mathrm{y}$ & $d$ & & 5,19 & $\mathrm{ma}$ & $\mathrm{w}$ & $\mathrm{d}$ \\
\hline Platypus sp. & & & & & & 0,04 & $\mathrm{r}$ & $\mathrm{z}$ & nd \\
\hline Scolytidae & & & & & & & & & \\
\hline Amphicranus sp. & 0,02 & $r$ & $\mathrm{z}$ & nd & & & & & \\
\hline Corthylus nudipennis & & & & & & 0,04 & $\mathrm{r}$ & $\mathrm{z}$ & nd \\
\hline Scolytidae & & & & & & & & & \\
\hline Corthylocurus sp. & & & & & & 0,04 & $\mathrm{r}$ & $\mathrm{z}$ & nd \\
\hline Cnesimus dryographus & & & & & & 0,07 & $\mathrm{r}$ & $\mathrm{z}$ & nd \\
\hline Cnesinus sp. & 0,02 & $\mathrm{r}$ & $\mathrm{z}$ & nd & & & & & \\
\hline Cryptocarenus diadematus & 15,29 & ma & w & $\mathrm{d}$ & & 16,43 & $\mathrm{ma}$ & w & d \\
\hline Cryptocarenus heveae & 21,06 & $\mathrm{ma}$ & $w$ & $\mathrm{~d}$ & & 13,44 & $\mathrm{ma}$ & w & d \\
\hline Cryptocarenus seriatus & 18,64 & $\mathrm{ma}$ & w & $\mathrm{d}$ & & 19,77 & $\mathrm{ma}$ & w & $\mathrm{d}$ \\
\hline Cryptocarenus sp. & 0,07 & $\mathrm{r}$ & $\mathrm{z}$ & nd & & 1,83 & $\mathrm{c}$ & $\mathrm{y}$ & d \\
\hline Hypothenemus bolivianus & 1,19 & $\mathrm{c}$ & $\mathrm{z}$ & $d$ & & 0,23 & $\mathrm{~d}$ & $\mathrm{z}$ & $\mathrm{d}$ \\
\hline Hypothenemus elephas & 0,22 & $\mathrm{~d}$ & $\mathrm{z}$ & $\mathrm{d}$ & & 0,29 & $\mathrm{~d}$ & $\mathrm{z}$ & $\mathrm{d}$ \\
\hline Hypothenemus eruditus & 5,36 & $\mathrm{ma}$ & w & $\mathrm{d}$ & & 3,29 & $\mathrm{ma}$ & w & $\mathrm{d}$ \\
\hline Hypothenemus obscurus & 13,83 & $\mathrm{ma}$ & w & $\mathrm{d}$ & & 10,62 & $\mathrm{ma}$ & w & $\mathrm{d}$ \\
\hline Hypothenemus sp. 1 & 1,29 & $\mathrm{c}$ & $\mathrm{z}$ & d & & 0,78 & $\mathrm{~d}$ & $\mathrm{z}$ & $\mathrm{d}$ \\
\hline Hypothenemus sp. 2 & 3,52 & $\mathrm{c}$ & $\mathrm{y}$ & $\mathrm{d}$ & & 1,56 & $\mathrm{c}$ & $\mathrm{y}$ & $\mathrm{d}$ \\
\hline Hypothenemus sp.3 & 0,12 & $\mathrm{r}$ & $\mathrm{z}$ & nd & & 0,01 & $\mathrm{r}$ & $\mathrm{z}$ & nd \\
\hline Microcorthylus minimus & & & & & & 0,41 & $\mathrm{c}$ & $\mathrm{z}$ & $\mathrm{d}$ \\
\hline Microcorthylus sp. & 0,02 & $\mathrm{r}$ & $\mathrm{z}$ & nd & & & & & \\
\hline Monarthum glabriculum & & & & & & 0,01 & $\mathrm{r}$ & $\mathrm{z}$ & nd \\
\hline Monarthum sp. & 0,02 & $\mathbf{r}$ & $\mathrm{z}$ & nd & & & & & \\
\hline Premnobius cavipennis & 0,41 & $\mathrm{r}$ & $\mathrm{z}$ & $\mathrm{d}$ & & 0,97 & $\mathrm{~d}$ & $\mathrm{y}$ & $\mathrm{d}$ \\
\hline Sampsonius dampfi & & & & & & 0,23 & $\mathrm{~d}$ & $\mathrm{z}$ & $\mathrm{d}$ \\
\hline Tricolus pernanulus & & & & & & 0,01 & $\mathrm{r}$ & $\mathrm{x}$ & nd \\
\hline Tricolus sp. & 0,02 & $\mathrm{r}$ & $\mathrm{z}$ & nd & & & & & \\
\hline Xyleborus affinis & 0,78 & $\mathrm{c}$ & $\mathrm{z}$ & $\mathrm{d}$ & & 1,67 & $\mathrm{~d}$ & $\mathrm{y}$ & $\mathrm{d}$ \\
\hline Xyleborus brasiliensis & 0,05 & $\mathrm{r}$ & $\mathrm{z}$ & nd & & 0,04 & $\mathrm{r}$ & $\mathrm{z}$ & nd \\
\hline Xyleborus bisseriatus & & & & & & 0,01 & $\mathrm{r}$ & $\mathrm{z}$ & nd \\
\hline Xyleborus ferrugineus & 0,80 & $\mathrm{c}$ & $\mathrm{z}$ & $\mathrm{d}$ & & 2,65 & a & w & $\mathrm{d}$ \\
\hline Xyleborus gracillis & 0,02 & $\mathrm{r}$ & $\mathrm{z}$ & nd & & & & & \\
\hline Xyleborus hagedorni & 0,02 & $\mathrm{r}$ & $\mathrm{z}$ & nd & & & & & \\
\hline Xyleborus obtruncatus & & & & & & 0,01 & $\mathrm{r}$ & $\mathrm{z}$ & nd \\
\hline Xyleborus paraguayensis & & & & & & 0,01 & $\mathrm{r}$ & $\mathrm{z}$ & nd \\
\hline Xyleborus retusus & 5,54 & $\mathrm{ma}$ & w & $\mathrm{d}$ & & 2,11 & a & $\mathrm{y}$ & $\mathrm{d}$ \\
\hline Xyleborus sentosus & 0,02 & $\mathrm{r}$ & $\mathrm{z}$ & nd & & & & & \\
\hline Xyleborus spinosulus & 5,48 & $\mathrm{ma}$ & w & $\mathrm{d}$ & & 12,98 & ma & $\mathrm{w}$ & $d$ \\
\hline Xyleborus truncatelus & & & & & & 0,01 & $r$ & z & nd \\
\hline Xyleborus sp.1 & & & & & & 0,03 & $r$ & $\mathrm{z}$ & nd \\
\hline Xyleborus sp. 2 & & & & & & 0,01 & $\mathrm{r}$ & $\mathrm{z}$ & nd \\
\hline
\end{tabular}




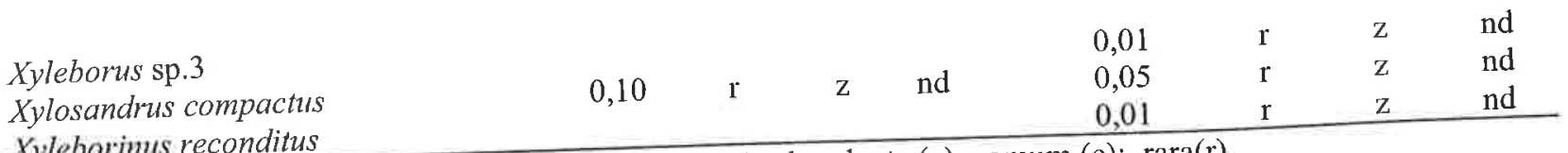

Xyleborimus reconditus

Freqüência (\%); Abundância (A): muito abundante (ma); abundante (a); comum (c); rara(r).

Constância (C): constante (w); acessória $(\mathrm{y})$; acidental $(\mathrm{z})$.

Dominância (D) :dominante (d); não dominante (nd).

Fazolin (1991) observou que dos 162 gêneros e espécies de insetos coletados em plantios de $H$. brasiliensis, $96,9 \%$ foram dominantes e sugeriu que o maior número de gêneros e espécies adaptou-se ao novo habitat e que as reduções verificadas posteriormente, neste número, podem ser atribuídas a capacidade de algumas espécies se tornarem modificadoras do ecossistema da seringueira, a ponto de influenciarem no desaparecimento gradativo de outros táxons.

Nas áreas amostradas, os índices de diversidades foram muito próximos. Entretanto, no talhão de $C$. citriodora ocorreu maior diversidade, que se refletiu em melhor relação entre o número de espécies abundantes (N1) e o número de espécies muito abundantes (N2), indicando distribuição mais eqüitativa entre as espécies

de coleópteros dentro desta área, com baixa predominância de espécies muito abundantes e melhor distribuição das populações ao longo do período de coletas (Tabela 5). Os resultados obtidos por Chey et al. (1997) em levantamento populacional de mariposas em vários ambientes, incluindo área com vegetação nativa, mostraram que na área com Eucalyptus spp. ocorreu maior índice de riqueza e abundância de espécies de insetos e atribuíram esta alta diversidade, à permanência da vegetação nativa dentro dos talhões, que teria contribuído para o aumento da diversidade servindo de hospedeiros para muitas larvas de mariposas, que encontraram nos reflorestamentos condições ideais de desenvolvimento e abrigo contra inimigos naturais.

TABELA 5. Índice de diversidade de acordo com a série de números de Hill e equitatividade dos coleópteros capturados em armadilhas etanólicas em plantios de Eucalyptus citriodora e de Eucalyptus urophylla. Cuiabá, MT, março de 1998 a fevereiro/1999.

\begin{tabular}{lcccc} 
Eucalyptus urophylla. Cuiaba, MT, março de 1998 a & \multicolumn{4}{c}{ Indice de Diversidade } \\
\cline { 2 - 5 } & $\mathrm{N} 0$ & $\mathrm{~N} 1$ & $\mathrm{~N} 2$ & $\mathrm{E}$ \\
Espécies & 43 & 10,45 & 7,53 & 0,69 \\
\hline Eucalyptus citriodora & 59 & 11,95 & 8,42 & 0,67 \\
Eucalyptus urophylla & & &
\end{tabular}

N0 = número total de espécies;

$\mathrm{N} 1=$ número de espécies abundantes;

$\mathrm{N} 2$ = número de espécies muito abundantes;

$\mathrm{E}=$ equitatividade entre as espécies.

$O$ local da pesquisa apresentou condições climáticas bem definidas, sem ocorrência de grandes variações ao longo dos últimos anos, com a ausência de invernos rigorosos de longos períodos de estiagem e de altas temperaturas. Os estudos das flutuações populacionais demonstraram que a maioria das espécies apresentou elevação nos números de indivíduos coletados, nos meses de baixa precipitação pluvial.

No talhão de $E$. citriodora, a população de C. seriatus apresentou picos populacionais em junho e outubro (Figura 1) e correlacionou-se negativamente com a umidade relativa (Tabela 6).

Esta correlação diverge dos resultados encontrados por Dorval (2002) que observou em talhão de E. pellita, pico 
populacional desta espécie no mês de outubro e a não correlação significativa com nenhum dos elementos climáticos.

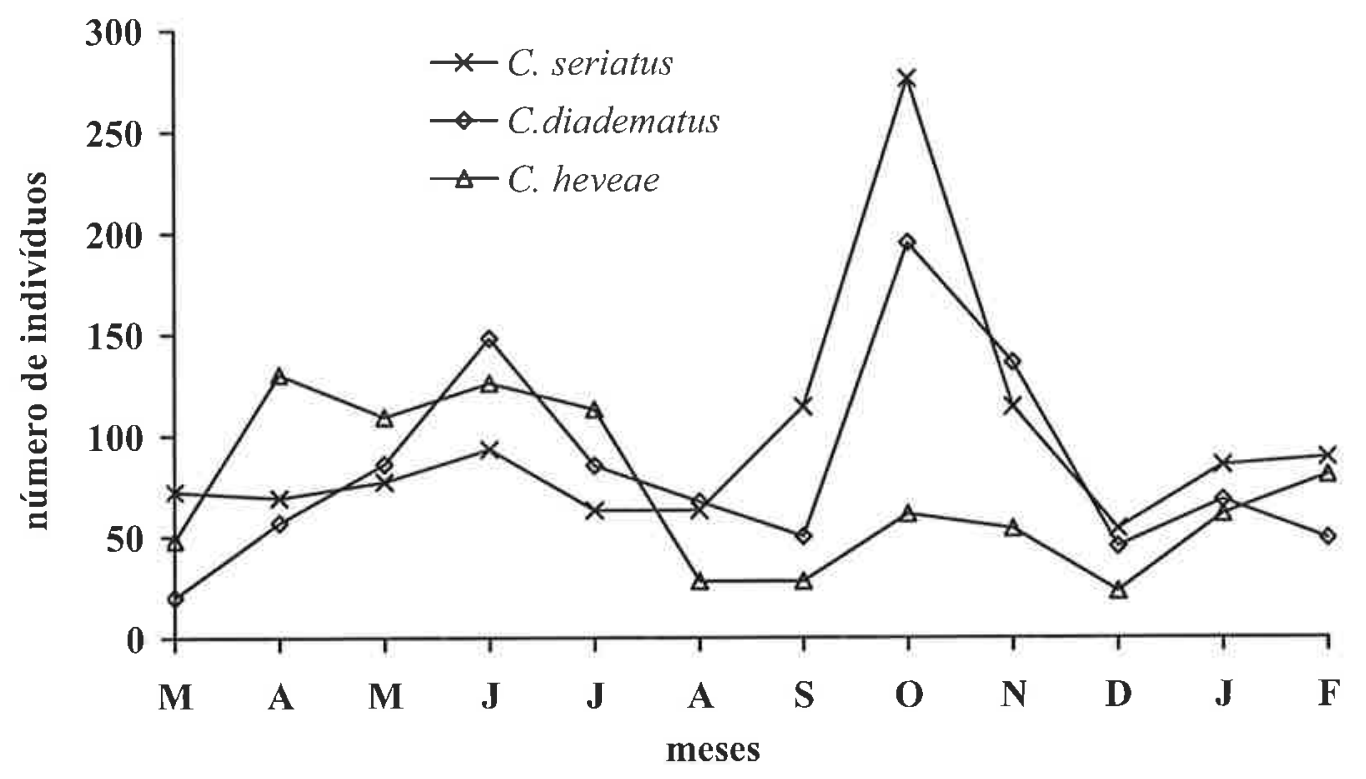

FIGURA 1. Flutuação populacional de Cryptocarenus seriatus, Cryptocarenus diadematus e Cryptocarenus heveae em talhão de Eucalyptus citriodora. Cuiabá, MT, março/ 1998 a fevereiro/1999.

TABELA 6. Correlações entre as espécies de coleópteros coletadas nos talhões de Eucalyptus citriodora e de Eucalyptus urophylla e as temperaturas mínima (TMI), média (TM), máxima (TMA), umidade relativa (UR) e precipitação pluvial (PP).Cuiabá, MT, março/1998 a fevereiro/1999.

\begin{tabular}{|c|c|c|c|c|c|c|c|c|c|c|}
\hline \multirow[b]{2}{*}{ Espécies } & \multicolumn{5}{|c|}{ Eucalyptus citriodora } & \multicolumn{5}{|c|}{ Eucalyptus urophylla } \\
\hline & TMI & TM & TMA & UR & $\mathrm{PP}$ & TMI & TM & TMA & UR & $\mathrm{PP}$ \\
\hline Cryptocaremus diadematus & $-\mathrm{S}^{* *}$ & NS & NS & NS & $-\mathrm{S}^{* *}$ & NS & $-S^{*}$ & NS & NS & NS \\
\hline Cryptocarenus heveae & NS & NS & NS & NS & $-\mathrm{S}^{* *}$ & NS & $-S^{*}$ & NS & NS & NS \\
\hline Cryptocarenus seriatus & NS & NS & NS & $-S^{* *}$ & NS & NS & NS & NS & NS & NS \\
\hline Hypothenemus eruditus & NS & NS & NS & NS & NS & NS & NS & NS & NS & NS \\
\hline Hypothenemus obscurus & NS & NS & NS & NS & NS & $+\mathrm{S}^{* *}$ & $+\mathrm{S}^{* *}$ & NS & NS & $+\mathrm{S}^{* *}$ \\
\hline Xyleborus retusus & NS & NS & NS & NS & NS & NS & NS & NS & NS & NS \\
\hline Xyleborus spinosulus & NS & NS & NS & NS & NS & NS & NS & NS & NS & NS \\
\hline Platypus linearis & NS & NS & NS & NS & NS & NS & NS & NS & NS & NS \\
\hline
\end{tabular}

$\mathrm{S}=$ Correlação significativa; $\mathrm{NS}=$ correlação não significativa.
$\left(^{*}\right)=$ significativa ao nivel de $5 \%$ de probabilidade.
$(* *)=$ Correlação significativa ao nível de $1 \%$ de probabilidade.
$(+)=$ correlação positiva.
$(-)=$ correlação negativa. 
camaldulensis picos populacionais da espécie em abril e fevereiro. Esta espécie não apresentou correlação significativa com nenhum dos elementos climáticos (Tabela 6).

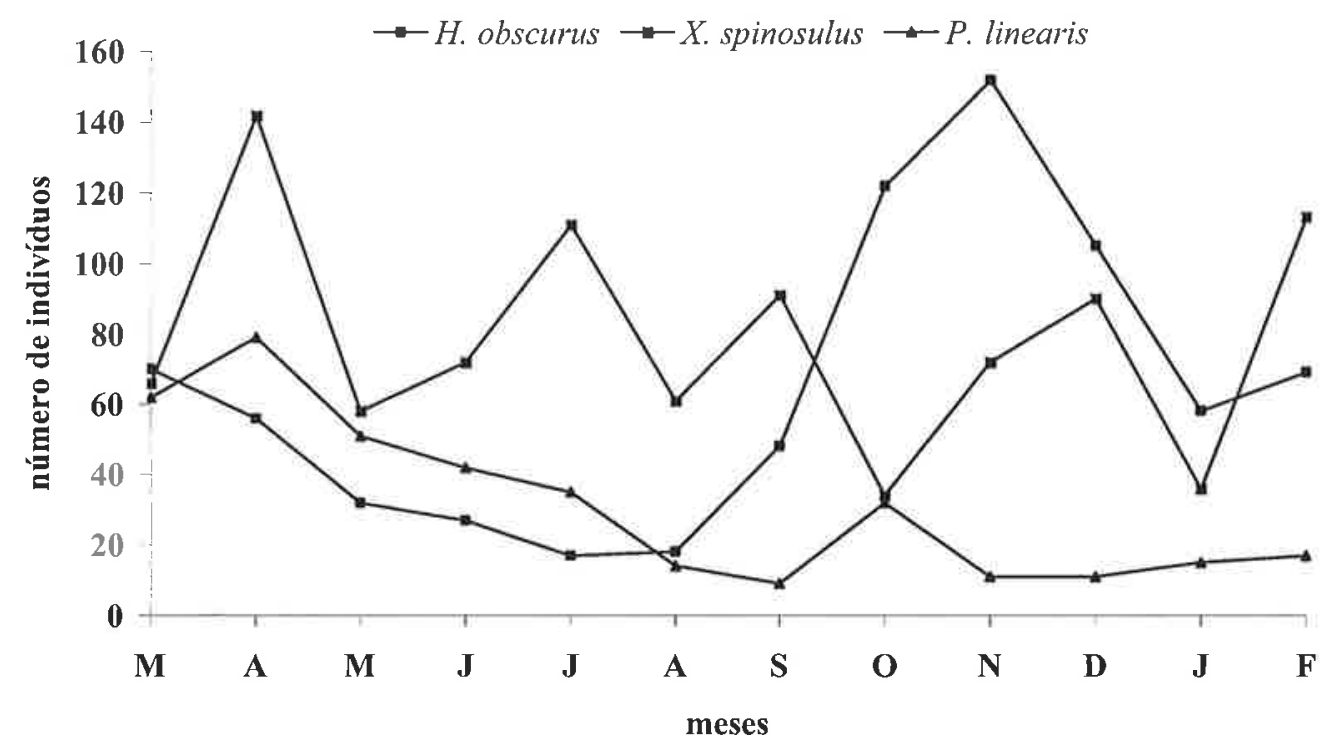

FIGURA 4. Flutuação populacional de Hypothenemus obscurus, Xyleborus spinosulus e Platypus linearis em talhão de Eucalyptus urophylla. Cuiabá, MT, março/1998 a fevereiro/1999.

\section{CONCLUSÕES}

Scolytidae é a família mais representativa nos talhões das duas espécies de eucaliptos;

No talhão de Eucalyptus citriodora, quantitativamente, as espécies Cryptocarenus heveae e $C$. seriatus (Scolytidae) são as mais importantes;

No talhão de Eucalyptus urophylla, quantitativamente, Cryptocarenus diadematus e $C$. seriatus (Scolytidae) são as mais representativas.

No talhão de Eucalyptus urophylla é coletado maior quantidade de espécies e de indivíduos;

No talhão de Eucalyptus urophylla maior quantidade espécies são constantes, muito abundantes e dominantes.
O talhão de Eucalyptus citriodora apresenta melhor eqüitatividade entre as espécies de insetos.

Nos talhões das duas espécies de eucaliptos, as espécies estudadas apresentam maior quantidade de picos populacionais nos meses de baixa precipitação pluvial.

\section{AGRADECIMENTOS}

À empresa Sadia Frigobrás Indústria e Comércio S.A e aos técnicos Neodir Crozzeta e Manoel Lauro da Silva pela colaboração na instalação e desenvolvimento deste projeto.

\section{REFERÊNCIAS BIBLIOGRÁFICAS}

BERTI FILHO, E. Impacto de Coleoptera; Cerambycidae em florestas de 
Eucalyptus no Brasil. Scientia Forestalis, Piracicaba, v. 52, p. 51- 54 p. 1997.

BIGGER, M. The insect pest of Eucalyptus deglupta in the Solomon Island. In: IUFRO. WP S2. 07.07 - PROTECION OF FORESTS IN THE TROPICS, 1982, Curitiba. Anais... Universidade Federal do Paraná, 1982, p. 142- 46.

BODENHEIMER, F.S. Precis d'ecologie animale, Paris, Payot, 1955

BROWNE, F.G.; HARRIS, A.; CAMPBELL, K.G.; WRIGHT, G. McK. Ecological studies in the borer horizontal of Austroplatypus incompertus (Schedl) (Coleoptera: Platypodidae). Diary of the Entomological society of Australia, Qweesland N.S.W. v. 6, n. 9, p. 11-21, 1976.

CHARARAS, C.; CHIPUILET, J. M. Studies on the digestion of cellulose by the larvae of the Eucalyptus borer, Phoracantha semipunctata (Coleoptera: Cerambycidae). Aust. J. Biol. Sci., Austrália, v. 36, p. 223-33, 1983.

CHEY, V.K.; HOLLOWAY, J.D.; SPEIGHT, M.R. Diversity of moths in forest plantations and natural forests in Sabah. Bulletin of Entomological Research, London, v. 87, p. 371-85, 1997.

DALL'OGLIO, O. T.; PERES FILHO, O. Levantamento e flutuação populacional de coleobrocas em plantios homogêneos de seringueira em Itiquira-MT. Scientia Forestalis, Piracicaba, v. 51, p. 49-58, 1997.

DAJOZ, R. Tratado de ecologia. Madrid: Mundi, 1974. 478 p.

DISPERATI, A. A. Sensoriamento remoto para a detecção, mapeamento e monitoramento dos danos causados por pragas florestais. Curitiba, FUPEF, $1995,40 \mathrm{p}$.
DORVAL, A. Levantamento populacional de coleópteros com armadilhas etanólicas em plantios de Eucalyptus spp. em uma área com vegetação de cerrado no município de Cuiabá, estado de Mato Grosso. Curitiba, 2002. 141p. Tese (Doutorado em Ciências Biológicas), Universidade Federal do Paraná, UFPr. Curitiba.

DORVAL, A.; PERES FILHO, O. Levantamento e flutuação populacional de coleópteros em vegetação de cerrado da baixada cuiabana, MT. Ciência Florestal, Santa Maria, v.11, n. 2, p. $171-182,2001$.

FAZOLIN, M. Análise Faunística de insetos coletados com armadilha luminosa em seringueira no Acre. Piracicaba, 1991. 237 f. Tese (Doutorado em Ciências), Departamento de Entomologia, Escola Superior de Agricultura "Luiz de Queiróz", Universidade de São Paulo.

FLETCHMANN, C. A.H.; GASPARETO, C.L. Scolytidae em pátio de serraria da fábrica Paula Souza (Botucatu/SP) e fazenda Rio Claro (Lençóis Paulista/SP). Scientia Forestalis, Piracicaba, n. 51, p. 61-75, 1997.

GRAY, B. Economic tropical forest entomology. An. Rev. Entomology, Stanford, v. 17, p. 313- 354. 1972.

HAWKESWOOD, T.J. New larval host registrations goes eight Australian jewel beetles (Coleoptera, Buprestidae). Giornale Italiani di Entomologia, Itália, v. 3, p. 173-177, 1986.

HOCKEY, M.J. Eucalyptus maculata Hook (Myrtaceae), a new host registered for Calymmaderus incisus Lea (Coleoptera:Anobiidae). AustralianEntomological-Magazine, Austrália, v. 12, p. 6-114, 1986.

JACOBONI, A. Due coleotteri dannosi agli eucaliptus.

Informatore 
Fitopatologico, Itália, v. 32, n. 6, p. 27-30, 1982.

LUDWIG, J.C.; REYNOLDS, J.F. Statistical ecology: a primer on methods and computing. New York, Wiley Interscience Publication, 1988. $337 \mathrm{p}$.

MACEDO, N. Estudo das principais pragas das ordens Lepidoptera e Coleoptera dos eucaliptais do estado de São Paulo. Piracicaba. 1975. 85 f. Dissertação (Mestrado em Entomologia). Escola Superior de Agronomia "Luiz de Queiróz".

MAYORGA, S.I.; LANFRANCO, D.; PEREDO, H.; RUIZ, C.; VIVES, L. Escarabajos de corteza y mancha azul: Situación en Chile. IPEF, Piracicaba, v. 13, n. 33, p. 57-66, 2000.

MORAES, G. J.; BERTI FILHO, E. Coleobrocas que ocorrem em essências florestais. IPEF, Piracicaba, v. 16, p. 27- 42, 1974.

OHMART,C.P.; EDWARDS, P.B. Insect herbivory on Eucalyptus. An. Rev. of Entomology, Stanford, v. 36, p. $637-$ 657, 1991.

PINHEIRO, J.V. Contribuição para o conhecimento de insetos dos eucaliptais no Brasil. Anuário Brasileiro de Economia Florestal, v. 14, n. 14, p. 245-55, 1962.

ROBERTS, E.C. Forest Insects of Nigéria with notes on their biology and distribution comonwealth Forest. Inst., Dep. Forestry, 1969, 206 p.

SAKAGAMI S.F.; MATSUMURA, E. Relative abundance, phenology and flower preference of andrenamid bee in Sapporo, North Japan (Hymenoptera, Apoidea). Jap. J. Ecol., Japão, v.16, n.6, p. 237-250, 1967.

SILVA, A.G.D.; GONÇALVES, C.R.; GALVÃO, D.M.; GONÇALVES, A.J.L.; SILVA, J.G. M.N.; SIMONETI, L. Quarto catálago dos insetos que vivem nas plantas do Brasil: seus parasitos e predadores. . Rio de Janeiro, Ministério da Agricultura: Fundação IBGE. Parte II, tomo $1,1968.622 \mathrm{p}$.

SILVEIRA NETO, S.; NAKANO, O.; BARBIN, D.; VILLA NOVA, N.A.. Manual de ecologia dos insetos, 15 ed.. São Paulo: Ceres, 1976.

SCRIVEN, G. T.; REEVES, E. L.; LUCK, R. F. Beetle from Australia threatens Eucalyptus. California Agriculture, p. 4-6. 1986.

ZANUNCIO, J.C.; BRAGANÇA, M.A.L.; LARANJEIRO, A.J.; FAGUNDES, M. Coleópteros associados à eucaliptocultura nas regiões de São Mateus e Aracruz, Espírito Santo. Rev. Ceres, Viçosa, v. 41, n. 22, p. 584-90, 1993. 